\title{
Prospects for Electroweak Precision Measurements and Triple Gauge Couplings at a Staged ILC
}

\author{
Robert Karl* \\ Deutsches Elektronen-Synchrotron (DESY) and University of Hamburg \\ on behalf of the ILD concept group \\ E-mail: robert.karledesy.de
}

In the absence of a direct discovery of new particles, precision measurements of the properties of known particles will provide the most powerful probe for phenomena beyond the Standard Model. Future electron positron linear colliders with polarised beams, like the International Linear Collider (ILC), will provide a unique laboratory for such measurements, complementary to hadron colliders.

In this contribution, we will review in particular the prospects for electroweak precision measurements, like the left-right asymmetry, as well as for measurements of charged triple gauge couplings, based on simulations of the ILD detector concept for the ILC. In all of these, the exact knowledge of the beam polarisation and the beam energy plays an important role. Therefore we will also discuss the precision determination of these accelerator parameters from collision data. We will pay special tribute to the most recent discussions concerning a possible first stage of the ILC operating at a center-of-mass energy of 250 or $350 \mathrm{GeV}$, but also the full ILC running plan.

EPS-HEP 2017, European Physical Society conference on High Energy Physics 5-12 July 2017

Venice, Italy

${ }^{*}$ Speaker. 


\section{Introduction}

At lepton colliders, the initial state of the colliding particles is fully defined, including the orientation of their spins with respect to momenta which is called polarization. This enables high precision studies of known particles like the Higgs boson or the top quark and a rich program of searches for the new particles, which is complementary to searches at the LHC.

The International Linear Collider (ILC) [1] is a planned electron-positron collider with center-ofmass energies of up to $500 \mathrm{GeV}$ and a possible upgrade to $1 \mathrm{TeV}$. Its construction is under political consideration in the Kitakami region in the prefecture Iwate in Japan. At the ILC, both beams will be longitudinally polarized, $|80 \%|$ for $e^{-}$-beam and $|30-60 \%|$ for $e^{+}$-beam, including an individual switch of the polarization sign (helicity reversal) for both beams. This provides a choice between different spin configurations, which allows accurate measurements of the chiral structure of the electroweak interaction and possible new phenomena, e.g. Dark Matter.

The International Large Detector (ILD) is a concept for a detector at the ILC. It is designed as a multi-purpose detector and optimized with a clear view on precision physics program. This sets strong requirements on the ILD performance: A point resolution of the vertex detector better than $3 \mu \mathrm{m}$, a high tracking performance corresponding to the transverse momentum resolution of $\sigma_{1 / p_{T}} \approx 2 \cdot 10^{-5} \mathrm{GeV}^{-1}$ and a jet-energy resolution of $\sigma_{E} / E \approx 3-4 \%$ which allows a separation of hadronic decays from $Z, W$ and Higgs bosons. All studies were performed within the context of the ILD.

\section{Beam Polarization Measurement}

The usage of polarized beams provides great advantages for the ILC. It allows deep insights into the chiral structure of the weak-interaction for known and unknown particles as well as a sensitivity to additional EW observables (e.g. left-right-asymmetry) to physics beyond the Standard Model. Furthermore, with polarized beams it is possible to modify signal and background crosssections thus improving the significance.

However, since all event rates depend linearly on the polarization, it is important to provide a determination of the actual beam polarization at the permille-level in order to fully exploit the physics potential of the ILC [2]. This requirement can only be fulfilled by combining the fast timeresolved measurements of the laser-Compton polarimeters with an absolute scale calibration of the luminosity-weighted average polarization at the interaction point (IP) calculated from collision data.

Thus, the beam polarization will be measured online by two laser-Compton polarimeters $[3,4]$ per beam. Thereby, for each beam, the upstream polarimeter is located $1.65 \mathrm{~km}$ before the IP, while the downstream polarimeter is located $150 \mathrm{~m}$ after the IP. In order to determine the polarization at the IP, the result of the polarimeter measurement is extrapolated to the IP via spin tracking [5]. For the absolute scale calibration, the luminosity-weighed averaged polarization is additionally calculated from collision data providing the information directly at the IP.

This study will focus on the determination of the absolute scale calibration using the minimization between the measured cross section $\sigma_{\text {data }}$ and the theoretical expected cross section $\sigma_{\text {theory }}$ [6]. 
Thereby, the cross sections are defined by:

$$
\begin{aligned}
\sigma_{\text {data }} & :=\frac{D-B}{\varepsilon \cdot \mathscr{L}_{I}} \\
\sigma_{\text {theory }} & :=\frac{\left(1-P_{e^{-}}\right)}{2} \frac{\left(1+P_{e^{+}}\right)}{2} \cdot \sigma_{L R}+\frac{\left(1+P_{e^{-}}\right)}{2} \frac{\left(1-P_{e^{+}}\right)}{2} \cdot \sigma_{R L} \\
& +\frac{\left(1-P_{e^{-}}\right)}{2} \frac{\left(1-P_{e^{+}}\right)}{2} \cdot \sigma_{L L}+\frac{\left(1+P_{e^{-}}\right)}{2} \frac{\left(1+P_{e^{+}}\right)}{2} \cdot \sigma_{R R}
\end{aligned}
$$

With $D, B, \varepsilon, \mathscr{L}_{I}$ are the number of selected events, the expected number of background events, the detector selection efficiency and the integrated luminosity, respectively. $P_{e^{ \pm}}$is the beam polarization of the $e^{ \pm}$-beam and $\sigma_{L R}$ corresponds to the chiral cross section with purely left-handed electrons and right-handed positrons. The four chiral cross sections are calculated by WHIZARD [7] considering Initial State Radiation (ISR) and the ILC beam spectrum at the corresponding centerof-mass energy.

To maximize the precision on the luminosity-weighted averaged beam polarization, the Standard Model processes of interest should be characterized both by a large left-right asymmetry $A_{\mathrm{k}, 1}^{\mathrm{i}, \mathrm{j}}$ and by a large unpolarized cross-section $\sigma_{0}$, in order to boost measurement sensitivity to chiral structure of the underlying interaction as well as the statistical precision. $A_{\mathrm{k}, 1}^{\mathrm{i}, \mathrm{j}}$ and $\sigma_{0}$ are defined by:

$$
A_{\mathrm{k}, \mathrm{i}}^{\mathrm{i}, \mathrm{j}}:=\frac{\sigma_{i, j}-\sigma_{k, l}}{\sigma_{i, j}+\sigma_{k, l}} \quad \sigma_{0}:=\frac{1}{4} \sum \sigma_{i, j} \quad i, j, k, l \in\{L, R\} \quad i, j \neq k, l
$$

The values for $A_{\mathrm{RL}}^{\mathrm{LR}}$ and $\sigma_{0}$ for a few suitable processes can be found in Table 1. For illustration, only the left-right asymmetry $A_{\mathrm{RL}}^{\mathrm{LR}}$ was chosen in this study but in principle this approach would work similarly for all other left-right asymmetries ( eg. $A_{\mathrm{LL}}^{\mathrm{LR}}$, etc. ), as well. As can be seen from Table 1, $W$-pair production has the largest sensitivity to the beam polarization, because at the tree-level it is only possible for left-handed electrons and right-handed positrons. Furthermore, it also has a sufficiently large unpolarized cross section so that the precision on the beam polarization is not dominated by the statistical precision considering the integrated luminosity at the ILC. As seen in Table 1, the s-channel $Z$ exchange has a far lower sensitivity to the actual chiral structure. But calculations show that, due to the far larger unpolarized cross section, the sensitivity to the beam polarization is comparable to the $W$-pair production. The beam polarization can also be simultaneously determined from multiple processes including different channels in order to increase its precision.

\begin{tabular}{|l|c|c|}
\hline Process $[250 \mathrm{GeV}]$ & $A_{\mathrm{RL}}^{\mathrm{LR}}$ & $\sigma_{0}[\mathrm{pb}]$ \\
\hline$e^{+} e^{-} \rightarrow W^{+} W^{-} \rightarrow q \bar{q} l v$ & 0.982 & 4.74 \\
\hline$e^{+} e^{-} \rightarrow Z \rightarrow q \bar{q}$ & 0.289 & 50.1 \\
\hline$e^{+} e^{-} \rightarrow e^{-} \bar{v} W^{+} \rightarrow e^{-} \bar{v} q \bar{q}$ & 0.983 & 1.29 \\
\hline$e^{+} e^{-} \rightarrow e^{+} v W^{-} \rightarrow e^{+} v q \bar{q}$ & 0.983 & 1.29 \\
\hline$l=\mu, \tau ; \quad q=u, d, c, s, b$ & & \\
\hline
\end{tabular}

Table 1: A few processes with a large sensitivity to beam polarization and their corresponding values for the left-right asymmetry $A_{\mathrm{RL}}^{\mathrm{LR}}$ and the unpolarized cross section $\sigma_{0}$, defined in Eq. 2.3. 


\section{Simultaneous Determination of the Chiral Cross Section}

For the measurement of the luminosity-weighted averaged polarization from the total chiral cross sections, a precise knowledge of these cross sections is necessary. However, the beam polarization can also be determined from the chirality dependence of the differential cross section. Thus, by using differential cross section measurements, the beam polarization and the total chiral cross section can be determined simultaneously without relying on theoretical calculations alone. For the measurement of the total chiral cross sections the following parametrization was used: For each process and channel, a parameter $\alpha$ scales the unpolarized cross section and another parameter $\beta$ allows for a shift in the asymmetry. They are defined as:

$$
A \longrightarrow A+\beta \quad \sigma_{0} \longrightarrow \alpha \cdot \sigma_{0}
$$

A total of 14 standard model processes including their channels were used in this study. This results in a 32 parameter fit: four parameters for the beam polarization and two pseudo-nuisance parameters $\alpha, \beta$ per process and channel, as defined in Eq. 3.1. Thereby, all 28 pseudo-nuisance parameters are correctly determined without loss of precision on the beam polarization. The fit is performed with a $\chi^{2}$ over the Number of Degrees of Freedom (NDF) of $753.43 / 708$. The results for the statistical precision of all parameters can be found in Table 2. As it is shown, the statistical precision at a permille-level on all parameters is well achievable. Note that the results given in Table 2 come only from the statistical uncertainty of the beam polarization. A full concept of the implementation of systematic uncertainties can be found in [6].

Absolute Uncertainty of $P, \alpha$ and $\beta\left[10^{-4}\right]$

\begin{tabular}{|c|c|c|c|c|c|c|c|}
\hline$\Delta P_{e^{-}}^{-}$ & 3.4 & $\Delta P_{e^{-}}^{+}$ & 1.3 & $\Delta P_{e^{+}}^{+}$ & 3.9 & $\Delta P_{e^{+}}^{-}$ & 2.9 \\
\hline$\Delta \alpha_{W^{+}}(e v l v)$ & 12 & $\Delta \alpha_{W^{-}}(e v l v)$ & 12 & $\Delta \beta_{W^{-}}(e v l v)$ & 13 & $\Delta \beta_{W^{+}}(e v l v)$ & 6.2 \\
\hline$\Delta \alpha_{W^{+}}(e v q \bar{q})$ & 7.5 & $\Delta \alpha_{W^{-}}(e v q \bar{q})$ & 7.5 & $\Delta \beta_{W^{-}}(e v q \bar{q})$ & 7.6 & $\Delta \beta_{W^{+}}(e v q \bar{q})$ & 3.6 \\
\hline$\Delta \alpha_{W W}(q \bar{q} q \bar{q})$ & 5.6 & $\Delta \alpha_{W W}(l v l v)$ & 12 & $\Delta \beta_{W W}(l v l v)$ & 4.9 & $\Delta \beta_{W W}(q \bar{q} q \bar{q})$ & 1.6 \\
\hline$\Delta \alpha_{W W}(l v q \bar{q})$ & 5.4 & $\Delta \alpha_{Z Z}(q \bar{q} q \bar{q})$ & 10 & $\Delta \beta_{Z Z}(q \bar{q} q \bar{q})$ & 12 & $\Delta \beta_{W W}(l v q \bar{q})$ & 1.4 \\
\hline$\Delta \alpha_{Z Z}(l l l l)$ & 28 & $\Delta \alpha_{Z Z}(l l q \bar{q})$ & 9.8 & $\Delta \beta_{Z Z}(l l q \bar{q})$ & 12 & $\Delta \beta_{Z Z}(l l l l)$ & 34 \\
\hline$\Delta \alpha_{Z Z W W}(q \bar{q} q \bar{q})$ & 5.8 & $\Delta \alpha_{Z Z W W}(l v l v)$ & 12 & $\Delta \beta_{Z Z W W}(l v l v)$ & 8.1 & $\Delta \beta_{Z Z W W}(q \bar{q} q \bar{q})$ & 2.9 \\
\hline$\Delta \alpha_{Z}(q \bar{q})$ & 1.6 & $\Delta \alpha_{Z}\left(l^{+} l^{-}\right)$ & 2.4 & $\Delta \beta_{Z}\left(l^{+} l^{-}\right)$ & 4.2 & $\Delta \beta_{Z}(q \bar{q})$ & 3.3 \\
\hline
\end{tabular}

Table 2: Results for the absolute uncertainty on the polarization and the pseudo nuisance parameters $\alpha, \beta$ in $10^{-4}$ from the simultaneous fit at $\sqrt{s}=250 \mathrm{GeV}$ and $\mathscr{L}_{I}=2000 \mathrm{fb}^{-1}$. The fit is performed with a $\chi^{2} / \mathrm{NDF}$ of 753.43 / 708 .

\section{Measurement of Triple Gauge Couplings (TGC) at an $e^{+} e^{-}$Collider}

Since leptons almost exclusively interact electroweakly, lepton colliders are very sensitive to the electroweak interaction. Thus, they are well suited to measure electroweak triple gauge couplings. However, the measurement of these couplings also has a large sensitivity to the actual beam polarization. Thus, the uncertainty of polarization measurement would significantly affect the precision of TGC determination and vice versa. Therefore, it is necessary to measure anomalous TGC 
and the beam polarization simultaneously. This can be achieved in a similar way as the simultaneous calculation of the total chiral cross section, described in Sec. 3.

For a center-of-mass energy of $500 \mathrm{GeV}$, the simultaneous measurement of the beam polarization and anomalous TGC was studied using $W$-pair production in the semileptonic channel [8]. The precision on the three anomalous TGCs $g_{1}^{Z}, \kappa_{\gamma}$ and $\lambda_{\gamma}$ were determined simultaneously within an Effective Field Theory (EFT) approximation including second order terms. This study was repeated at a center-of-mass energy of $1 \mathrm{TeV}$ [9]. Both studies were performed with a full detector simulation of the ILD detector concept.

These analyses provide two reference points for TGC precision at different center-of-mass energies. The goal is an approximation on the TGC precision at $250 \mathrm{GeV}$ by extrapolating the $500 \mathrm{GeV}$ results to $250 \mathrm{GeV}$. Therefore, three scaling factors were considered; the first is the statistical scaling $f_{\text {stat }}$ which is just given by $1 / \sqrt{N}$ and the second $f_{\text {theo }}$ is the actual sensitivity to the TGCs which is assumed to scale with $M_{W}^{2} / s$. The third scaling factor $f_{\text {det }}$ is related to the energy dependence of the detector acceptance. Such a factor is hard to predict, and thus it is determined by the comparison between the $500 \mathrm{GeV}$ and $1 \mathrm{TeV}$ results. The final scaling factors are shown in Eq. 4.1.

$$
\begin{array}{rlrl}
\Delta c_{i}(\sqrt{s}) & =\Delta c_{i}(500 \mathrm{GeV}) \cdot f_{\text {stat }}\left(\sqrt{s} ; \mathscr{L}_{I}\right) \cdot f_{\text {theo }}(\sqrt{s}) \cdot f_{\text {det }, i}(\sqrt{s}) \\
f_{\text {stat }}\left(\sqrt{s} ; \mathscr{L}_{I}\right) & =\sqrt{\frac{500 \mathrm{fb}^{-1} \cdot \sigma(500 \mathrm{GeV})}{\mathscr{L}_{I} \cdot \sigma(\sqrt{s})} ;} & & f_{\text {theo }}(\sqrt{s})=\frac{(500 \mathrm{GeV})^{2}}{s} \\
f_{\text {det }, \Delta g}(\sqrt{s}) & =0.85+\frac{0.15}{500 \mathrm{GeV}} \cdot \sqrt{s} ; & & f_{\text {det }, \Delta \lambda}(\sqrt{s})=0.63+\frac{0.37}{500 \mathrm{GeV}} \cdot \sqrt{s} \\
f_{\text {det }, \Delta \kappa}(\sqrt{s}) & =1 ; &
\end{array}
$$

The achievable precision of the TGC measurement is shown in Table 3. At the ILC, a subpermille-level on anomalous TGC precision can already be reached in the first stage of the ILC with $250 \mathrm{GeV}$. This is roughly 2 orders of magnitude better than the current best limit on anomalous TGCs, as shown in Fig. 1.

\begin{tabular}{|c|c||c|c|c|}
\hline \multicolumn{2}{|c||}{ ILD full simulation [8] } & \multicolumn{3}{c|}{ Extrapolations } \\
\hline$E_{\mathrm{CMS}}$ & $500 \mathrm{GeV}$ & $250 \mathrm{GeV}$ & $350 \mathrm{GeV}$ & $\mathrm{H}-20[10]$ \\
\hline$\Delta g_{1}^{Z}$ & $4.3 \cdot 10^{-4}$ & $8.1 \cdot 10^{-4}$ & $5.0 \cdot 10^{-4}$ & $5.0 \cdot 10^{-4}$ \\
\hline$\Delta \kappa_{\gamma}$ & $4.4 \cdot 10^{-4}$ & $9.6 \cdot 10^{-4}$ & $5.5 \cdot 10^{-4}$ & $5.7 \cdot 10^{-4}$ \\
\hline$\Delta \lambda_{\gamma}$ & $4.1 \cdot 10^{-4}$ & $7.8 \cdot 10^{-4}$ & $5.0 \cdot 10^{-4}$ & $5.1 \cdot 10^{-4}$ \\
\hline
\end{tabular}

Table 3: Achievable precision of the Triple Gauge Couplings measurement for $\mathscr{L}_{\mathrm{I}}=2 \mathrm{ab}^{-1}$ displayed for different center-of-mass energies and the full benchmark running scenario H-20 of the ILC. 

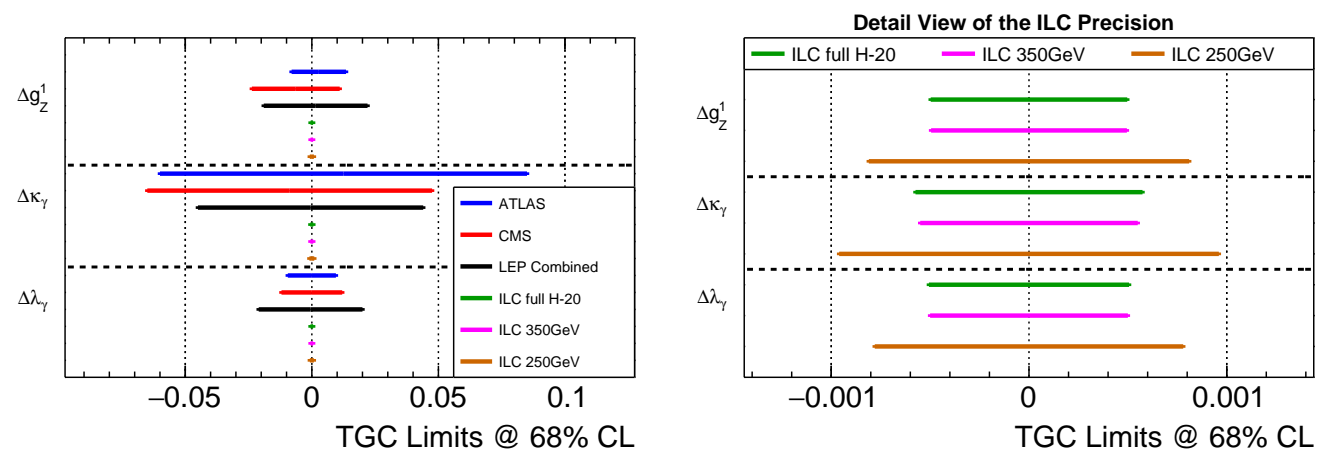

Figure 1: Comparison of the reachable TGC precision of the ILC, shown in Table 3, with the final results from LEP combined from ALEPH, L3 and OPAL results [11] and the LHC TGC limits for $\sqrt{s}=8 \mathrm{TeV}$ data and an integrated luminosity of $\mathscr{L}_{I}=20.3 \mathrm{fb}^{-1}$ and $\mathscr{L}_{I}=19.4 \mathrm{fb}^{-1}$ for ATLAS and CMS, respectively [12].

\section{References}

[1] ILC Technical Design Report, 2013, http://www.linearcollider.org/ILC/Publications/Technical-Design-Report Volume 1: "Executive Summary," [arXiv:1306.6327v1 [physics.ins-det]], Volume 2: "Physics," [arXiv:1306.6352v1 [physics.ins-det]], Volume 3.I: "Accelerator R\&D," [arXiv:1306.6353v1 [physics.acc-ph]], Volume 3.II: "Accelerator Baseline Design," [arXiv:1306.6328v1 [physics.acc-ph]], Volume 4: "Detectors," [arXiv:1306.6329v1 [physics.ins-det]].

[2] Annika Vauth, Jenny List. Beam Polarization at the ILC: Physics Case and Realization, Spin Physics (SPIN2014), International Journal of Modern Physics: Conference Series, 29 February 2016, http://www.worldscientific.com/doi/pdf/10.1142/S201019451660003X

[3] Jenny List, Annika Vauth, and Benedikt Vormwald: A Quartz Cherenkov Detector for Compton-Polarimetry at Future $e^{+} e^{-}$Colliders https://bib-pubdb1.desy.de/record/221054

[4] Jenny List, Annika Vauth, and Benedikt Vormwald: A Calibration System for Compton Polarimetry at $e^{+} e^{-}$Colliders https://bib-pubdb1.desy.de/record/289025

[5] Moritz Beckmann, Jenny List, Annika Vauth, and Benedikt Vormwald: Spin transport and polarimetry in the beam delivery system of the international linear collider http://iopscience.iop.org/article/10.1088/1748-0221/9/07/P07003/pdf

[6] Robert Karl, Jenny List, Polarimetry at the ILC, [arXiv:1703.00214]

[7] The WHIZARD Event Generator, A program system designed for the efficient calculation of multi-particle scattering cross sections and simulated event samples, https://whizard.hepforge.org/

[8] I. Marchesini, Triple Gauge Couplings and Polarization at the ILC and Leakage in a Highly Granular Calorimeter, PhD Thesis, University of Hamburg, 2011 [DESY-THESIS-2011-044], http://www-library.desy.de/preparch/desy/thesis/desy-thesis-11-044.pdf 
[9] Aura Rosca, Measurement of the charged triple gauge boson couplings at the ILC, Nuclear and Particle Physics Proceedings 273-275 (2016) 2226-2231, http://dx.doi.org/10.1016/j.nuclphysbps.2015.09.362

[10] Jenny List, Running Scenarios for the ILC,

LC Forum / Terascale Annual Meeting November 17-18, 2015, DESY http://pubdb.xfel.eu/record/289187/files/jlist_lcforum_1511.pdf?version=1

[11] ALEPH, DELPHI, L3, OPAL, and the LEP TGC Working Group, A combination of results on charged triple gauge boson couplings measured by the LEP experiments, LEPEWWG/TGC/2005-01.

[12] LHC limits on anomalous triple and quartic gauge couplings in comparison of the running experiments:

https://twiki.cern.ch/twiki/bin/view/CMSPublic/PhysicsResultsSMPaTGC 